\title{
Aplicación interuniversitaria del aprendizaje basado en retos en la asignatura de Mejora Genética Animal
}

Noelia Ibáñez Escrichea, Cristina Casto Rebollo ${ }^{a}$, Nora Formoso Rafferty ${ }^{\mathrm{b}}$, Oscar González Recio ${ }^{\mathrm{b}}$ y Joaquim Casellas Vidal ${ }^{\mathbf{c}}$

${ }^{a}$ Departamento de Ciencia Animal, Universitat Politècica de València, Camino de Vera. 14, València 46022, Spain; noeibes@dca.upv.es, criscasre@posgrado.upv.es ${ }^{b}$ Departamento de Producción Agraria, E.T.S.I.A.A.B, Universidad Politécnica de Madrid, C/ Senda del Rey s/n, 28040 Madrid, Spain; nora.formosorafferty@upm.es, oscar.grecio@upm.es ${ }^{c}$ Departamento de Ciencia Animal y de los Alimentos, Universitat Autònoma de Barcelona, Edifici V, Travessera dels Turons, 08193 Bellaterra, Barcelona; Joaquim.casellas@uab.cat.

\section{\$EWWFW}

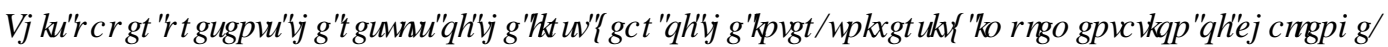
EDMHG ODDQQJ

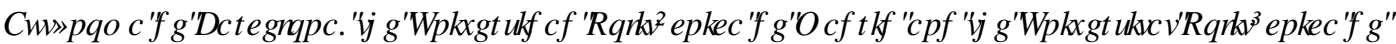

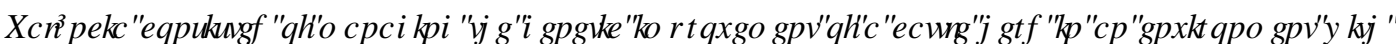

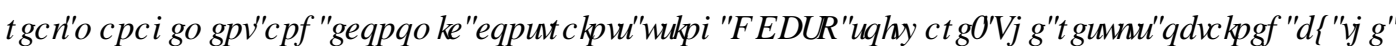

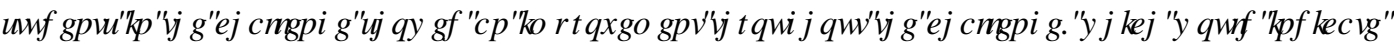

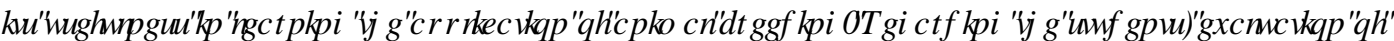

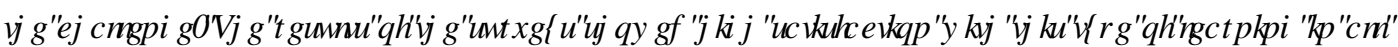

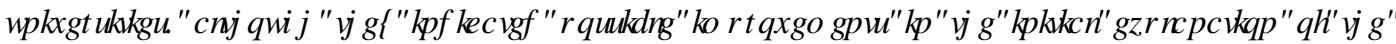

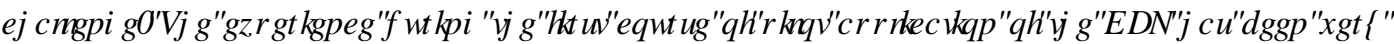

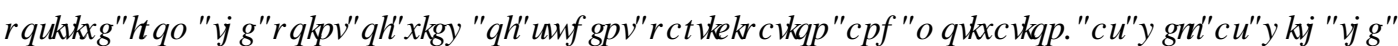

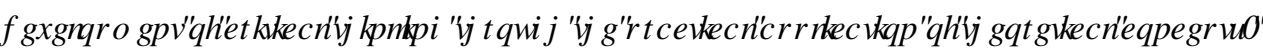

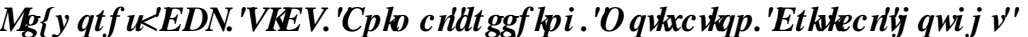

\section{HXP HQ}

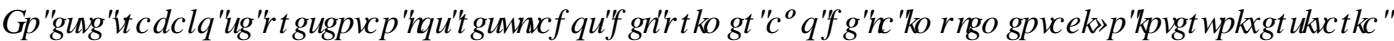

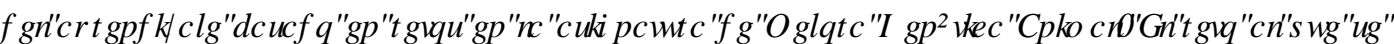

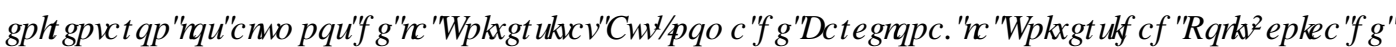

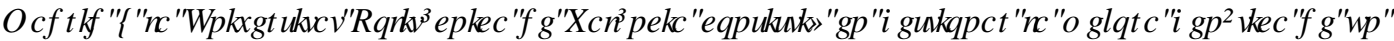

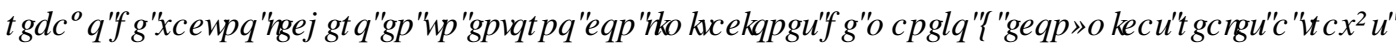

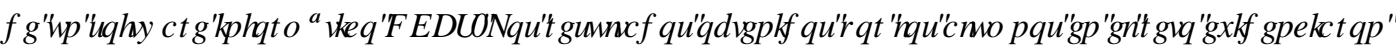

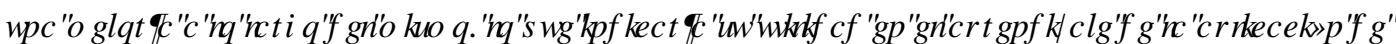

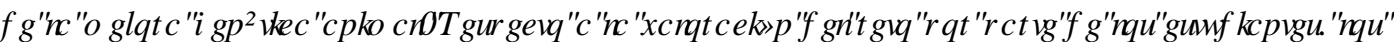

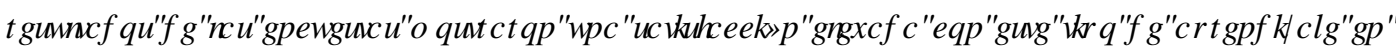

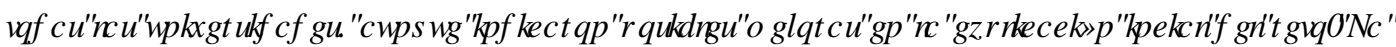

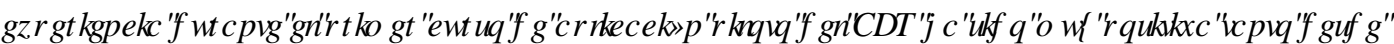

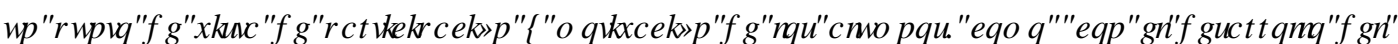

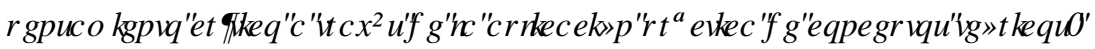

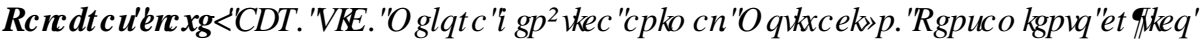




\section{Introducción}

La asignatura de Mejora Genética Animal se caracteriza por tener un fuerte contenido teórico y abstracto, centrado en las bases genéticas de la selección animal. Su potencial aplicación en especies tanto domésticas como salvajes implica conocimientos moderados de estadística, álgebra matricial y probabilidad, combinados con teoría genética, tanto clásica como contemporánea, y conceptos aplicados de producción animal. El objetivo de esta asignatura tal como describe Piedrafita (2015) es la formación de profesionales de la Mejora Genética Animal orientada a la adquisición de competencias específicas relacionadas con el diseño, organización y ejecución de Planes de Mejora. Sin embargo, la consecución de este objetivo requiere relacionar una gran diversidad de conocimientos de distintas áreas que se imparten en la asignatura. Hasta ahora, la metodología que se aplica habitualmente en las asignaturas de mejora genética animal está basada en clases magistrales y clases prácticas basadas en la resolución de ejercicios y pequeñas simulaciones de ordenador. Este tipo de metodologías tienen algunas limitaciones. Por una parte, las clases magistrales pueden inducir a la pasividad en el alumno, la dificultad para favorecer una asimilación inmediata de los contenidos y la limitación del pensamiento crítico y creativo del alumno (Beard y Hartley, 1984; Brown y Atkins, 1988). Por otra parte, la resolución de ejercicios y problemas ayudan a clarificar y consolidar los conocimientos adquiridos, pero su énfasis se enmarca en la comprensión del material de un texto y la elaboración de una solución numérica a problemas y no tanto a la resolución de casos reales. En cambio, en el aprendizaje basado en retos (ABR) se pide que los estudiantes aporten soluciones concretas a un problema complejo basado en el mundo real (Blank, 1997; Jou, Hung y Lai, 2010; Martí, 2010; Fletcher, 2011). El ABR es un ejercicio de gran ayuda para interiorizar los conceptos de la asignatura, ponerlos en práctica y reflexionar cómo la toma de decisiones influye en los resultados obtenidos. Sin embargo, la puesta en práctica de un caso real en mejora genética animal está condicionado a que la selección animal es un proceso lento, siendo el cambio de las características genéticas de las poblaciones animales a medio-largo plazo las que cobran interés dado al carácter acumulativo generación tras generación (Leopoldo, 2014).

\subsection{Aprendizaje basado en retos y tecnologías de la información y la comunicación.}

La simulación con ordenadores es una herramienta muy utilizada en el aprendizaje de la mejora genética animal (Carlson, 1985; Buchanan et al., 1988; Medrano et al., 2010). Sin embargo, hasta ahora, los programas de simulación existentes no abordaban los problemas reales en su complejidad, son simples y sin interactividad, por lo que no obligan a tomar decisiones de forma continua y autónoma por parte del estudiante. En los últimos años, el Dr. Joaquim Casellas de la Universitat Autònoma de Barcelona ha desarrollado un software de selección genética (http://www.casellas.info/dcbsp/dcbsp.php) donde el alumno se enfrenta al reto de llevar a cabo un programa de mejora real. En este software (DCBSP: Dairy Cattle Breeding Simulation Program), el alumno debe tomar todas las decisiones de selección de los animales de su rebaño en un entorno con limitaciones de manejo y económicas reales.

Las metodologías activas como el ABR requieren de una gran implicación por parte del profesor y el alumnado. Una de las grandes prácticas llevadas adelante en compañías exitosas para motivar a los empleados son los Sprint (Lang, 2017). Es decir, "carreras" que tienen como finalidad generar una competencia sana, a la vez que permiten a los empleados interiorizar nuevos conocimientos. Dentro de un aula, una competencia entre alumnos puede ser muy beneficiosa (Gavin y col., 2017). Es por ello, que, además de desarrollar el pensamiento crítico y el aprendizaje comprensivo, con este proyecto se pretenda potenciar la motivación del estudiante a través de dos estrategias:

1) Evaluar la evolución de cada programa de mejora llevado a cabo por el de alumno. 
2) Organizar un "sprint" interuniversitario donde no solo se compita con los alumnos de la clase sino entre diferentes universidades y grados.

La implantación de la segunda estrategia tiene como objetivo potenciar la motivación del alumno al permitirle competir semanalmente con alumnos de otras universidades y grados. La asignatura de mejora genética animal de la Universitat Politècnica de València (UPV) tiene pocos alumnos $(<20)$ con lo que la potencia para evaluar la eficacia de este tipo de enseñanza puede verse mermada. La aplicación interuniversitaria permite que el proyecto se transfiera a muchos más alumnos $(\sim 120)$ y que su eficacia en el aprendizaje se pueda evaluar correctamente.

\section{Objetivo}

El objetivo de este trabajo es analizar los resultados y la satisfacción del alumnado del primer año de implantación interuniversitaria del ABR, a través del programa de simulación DCBSP (Dairy Cattle Breeding Simulation Program) en las asignaturas de mejora genética animal del Grado de Genética de la Universitat Autònoma de Barcelona (UAB), del Grado en Ingeniería Agrícola de la Universidad Politécnica de Madrid (UPM), y del Grado Ingeniería Agroalimentaria y del Medio Rural en la intensificación de explotaciones agropecuarias de la UPV.

\section{Desarrollo de la innovación}

En el primer año de implementación del ABR se ha adaptado y rediseñado el sofware DCBSP para poder, simultáneamente, gestionar más de 150 rebaños. Una vez desarrollado el sofware se coordinó la implementación del reto, basado en este sofware, en las asignaturas de mejora genética animal de la UPV, UAB y UPM. Para ello, se fijó la aplicación del reto durante 6 semanas, de manera simultánea en las tres universidades, tiempo necesario para que los estudiantes evalúen ellos mismos las consecuencias de las decisiones tomadas y puedan, si es necesario, rectificarlas. Se elaboró un manual explicativo, común, del sofware DCBSP y un documento donde se especificaba claramente los objetivos del reto, calendario y sistema de evaluación. El reto al que se enfrentaban los alumnos consistía en aumentar el mérito genético de $\mathrm{kg}$ de leche producida en su rebaño, sin reducir el porcentaje de grasa contenida en la leche y sin aumentar la consanguinidad de los animales del rebaño más del 10\%. Además, es importante destacar que también se aplicaron dos restricciones adicionales, una técnica y otra económica:

- Técnica: En cada ronda semanal se deben asignar los toros de inseminación artificial a un mínimo de 40 vacas/terneras (el máximo son 99 inseminaciones).

- Económica: El presupuesto disponible en cada ronda semanal es de 2.000 euros. Los remanentes de presupuesto no se acumulan para la ronda siguiente.

Durante estas 6 semanas (correspondientes a 6 años virtuales) el alumnado llevó a cabo un programa de mejora real donde se utilizaba toda la tecnología disponible, es decir, desde los fenotipos y genealogía (selección tradicional) a marcadores moleculares (selección genómica). Cada estudiante disponía de su propio rebaño (conectado genealógicamente con el resto a través de los sementales disponibles) y tomó decisiones sobre sus animales del tipo: «qué vacas continúan en el rebaño y cuáles se descartan», «qué terneras tienen suficiente potencial genético para ser seleccionadas y promocionar el ganado adulto», y «con qué toro tengo que cruzar cada una de las vacas». De lunes a viernes, el alumno tuvo que reflexionar sobre estas preguntas, tomar las decisiones oportunas, e introducir la información telemáticamente. Durante el fin de semana, el programa se ejecutó de nuevo, integró toda la información proporcionada por los alumnos, e hizo evolucionar un año todos los rebaños, de manera virtual, proporcionando un nuevo conjunto de información actualizada de cada rebaño (Figura 1). Finalmente, los alumnos junto al profesor evaluaron 
los resultados (cada semana) derivados de las diferentes decisiones en términos de eficiencia de la selección efectuada en su programa y en comparación con los resultados obtenidos por el resto de los compañeros.

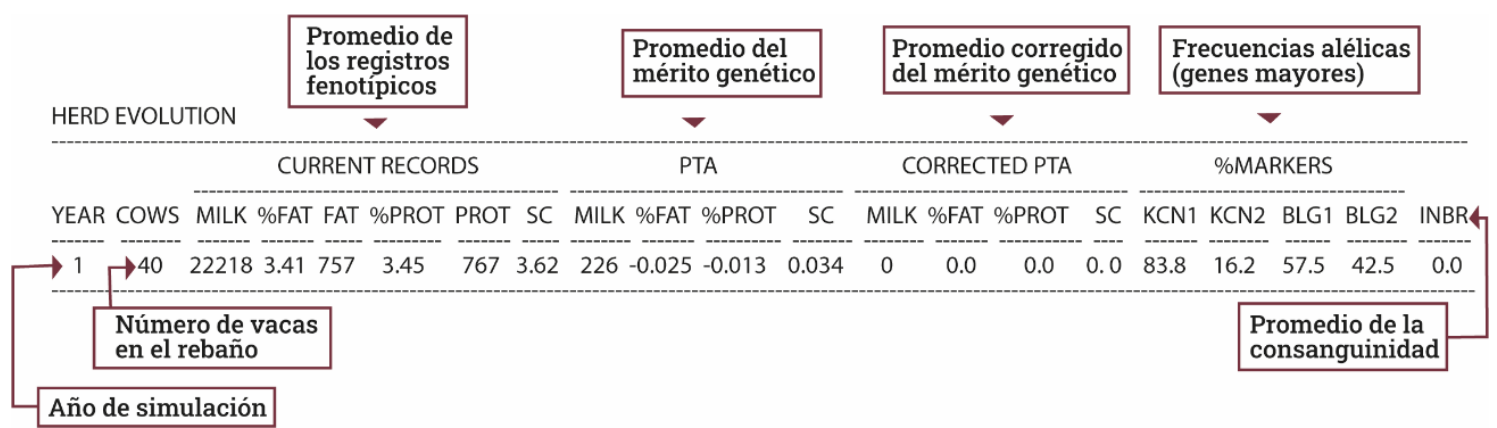

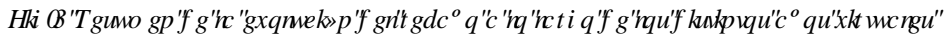

Los resultados para cada año virtual y rebaño fueron almacenados para evaluar posteriormente el impacto de la adquisición de conceptos de mejora genética en la evolución del reto, teniendo en cuenta posibles efectos de confusión como la Universidad o sexo en un modelo de regerión lineal. El efecto Universidad, sexo o año virtual de selección se testó con test de Fisher fiando el nivel de significación $\alpha=0.05$. Asi mismo, al final de las asignaturas de las tres Universidades se realizó una evaluación de la implementación del reto a través de una encuesta a los estudiantes (Tabla 1) y se testó si había diferencias entre Universidades.

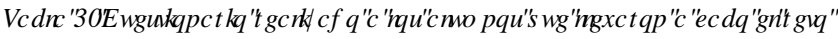

\begin{tabular}{|c|c|c|}
\hline Aspecto Valorado & Cuestionario & Puntuación \\
\hline \multirow{4}{*}{ Información Reto } & $\begin{array}{l}\text { Los objetivos quedaron claramente } \\
\text { definidos }\end{array}$ & $1-5$ \\
\hline & $\begin{array}{l}\text { Se especificó claramente la fecha de } \\
\text { inicio y final de la simulación }\end{array}$ & $1-5$ \\
\hline & $\begin{array}{l}\text { El sistema de evaluación fue } \\
\text { correctamente indicado }\end{array}$ & $1-5$ \\
\hline & $\begin{array}{l}\text { Explica el funcionamiento del } \\
\text { programa }\end{array}$ & $1-5$ \\
\hline \multirow[t]{4}{*}{ Manual } & Explica el contenido de las tablas & $1-5$ \\
\hline & $\begin{array}{l}\text { Indica donde se encuentra la } \\
\text { información relevante }\end{array}$ & $1-5$ \\
\hline & ¿Cómo de útil piensas que ha sido? & $1-5$ \\
\hline & ¿Te ha resultado complicado? & $1-5$ \\
\hline \multirow[t]{3}{*}{ Satisfacción ABR } & ¿Por qué? & Respuesta corta \\
\hline & Satisfacción general & $1-5$ \\
\hline & Satisfacción uso del programa & $1-5$ \\
\hline
\end{tabular}




\section{Aceptación}

¿Te gustaría repetirlo?

$1-5$

¿Obtendrías mejores resultados?

$1-5$

\section{Resultados}

En total 104 estudiantes, 12 del grado de la UPV, 32 de la UPM y 60 de la UAB participaron en el reto interuniversitario. Los resultados medios obtenidos para el mérito genético de $\mathrm{kg}$ de leche y \% de grasa en leche por semana (año virtual) para cada Universidad se muestran en la Figura 2. Se observa una evolución positiva en la producción de leche durante para todas las universidades y \% de grasa mayor de cero acorde con los objetivos planteados en el reto. Las diferencias en el éxito del reto entre universidades fueron relevantes solo entre UAB y UPM (p-valor =0.03). En relación al efecto sexo no se encontraron diferencias. Por otra parte, también se testó el éxito obtenido en las primeras dos semanas respecto a las dos últimas semanas. Los resultados mostraron diferencias relevantes entre ambos tramos (p-valor $<0.01$ ). Este resultado implicaría que hubo un aprendizaje en la aplicación de la mejora genética animal en el reto.
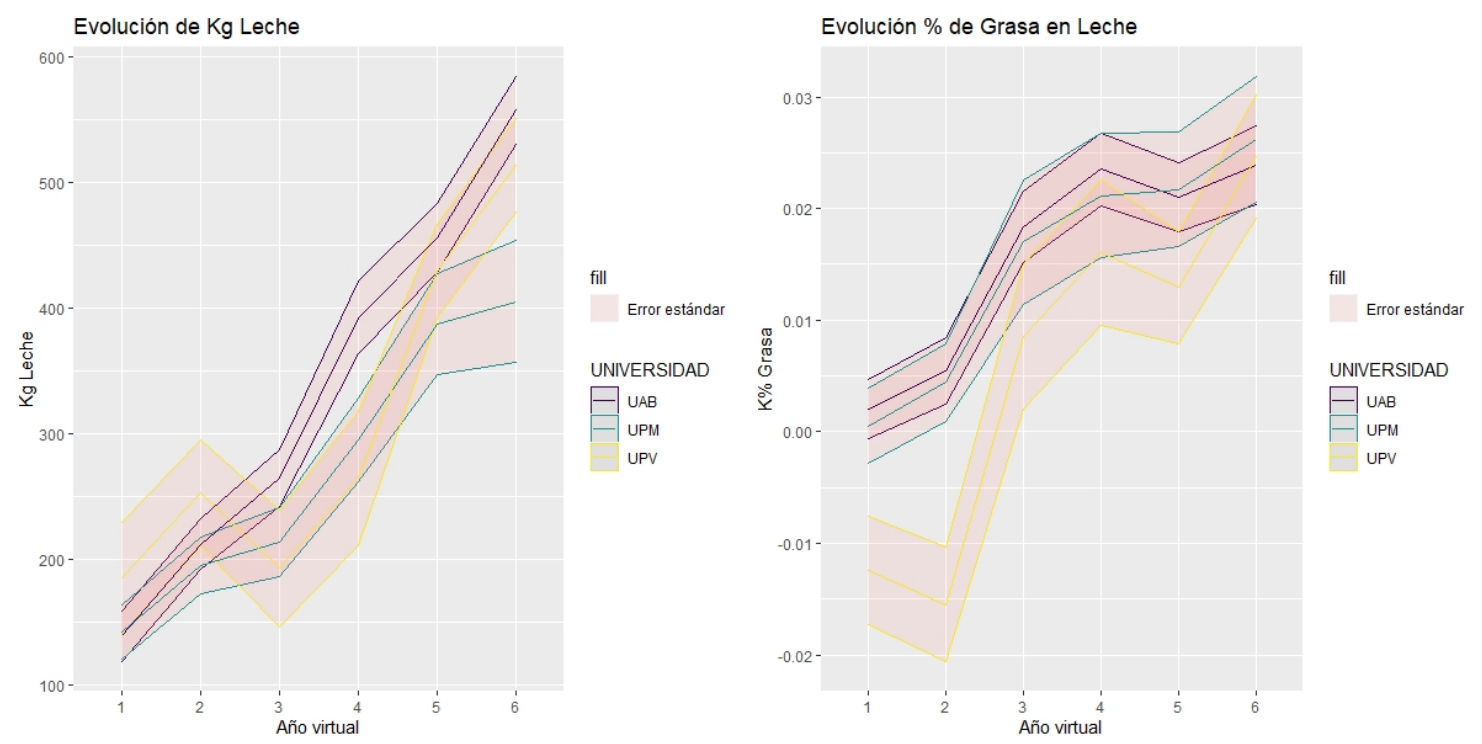

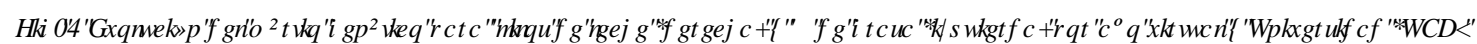

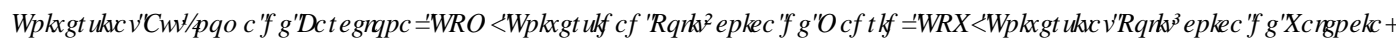

Un total de 41 encuestas fueron rellenadas por los estudiantes (12 UPV, 11 UPM y 18 UAB). La Figura 3 muestra la percepción que tienen los alumnos del manual de uso del sofware DCBSP y del documento donde se especificaba claramente los objetivos del reto, calendario y sistema de evaluación en el que se basa el ABR. . La puntuación media asignada fue de 3.9 sobre 5 a la explicación del contenido de las tablas, y un 3.8 sobre 5 a la explicación del funcionamiento del programa y al contenido de la información relevante para desarrollar correctamente el ABR. Estas valoraciones, cercanas a 4 sobre 5, indican una satisfacción global bastante buena en cuanto al manual desarrollado para el ABR, aunque nos alertan sobre la necesidad de mejorarlo. 


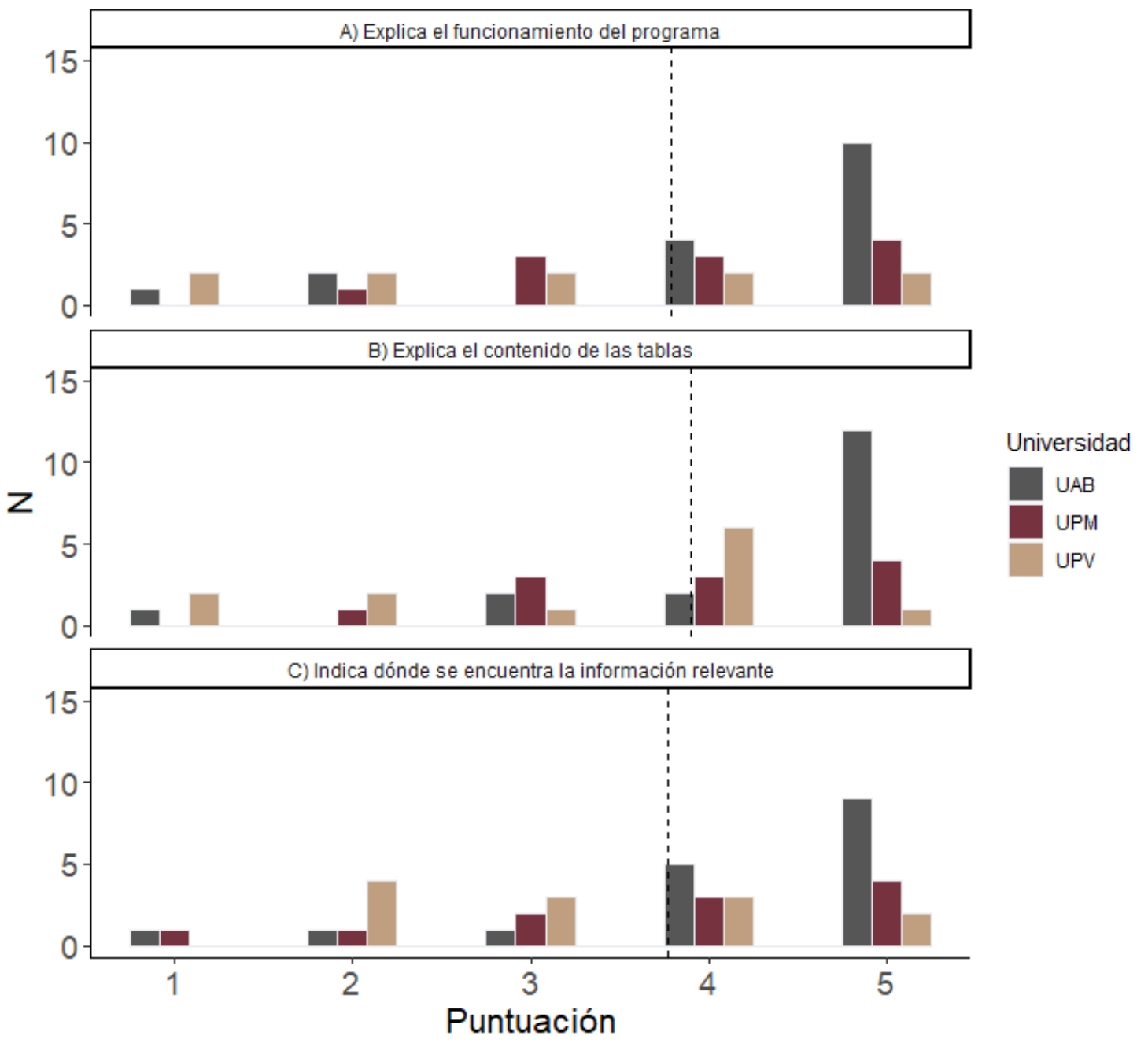

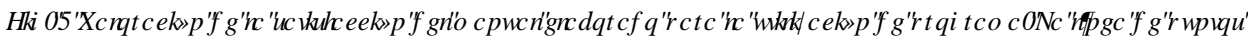

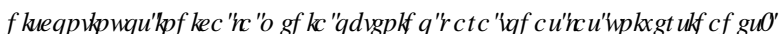

La Figura 4 muestra los resultados obtenidos para la valoración de la explicación de los aspectos importantes para llevar a cabo el ABR, como son los objetivos, las fechas de inicio y fin del mismo, y el sistema de evaluación a seguir. Los alumnos evaluaron muy positivamente, con un puntaje mayor a 4 sobre 5, la claridad con la que se marcaron las fechas de inicio y fin del ABR y su sistema de evaluación. Sin embargo, la explicación de los objetivos del ABR no fue valorada tan positivamente como se esperaba (3.7 sobre 5). Se supone que el factor determinante fue la dificultad de asociar los conceptos de las asignaturas con el ABR, ya que en todas las universidades este reto comienza al inicio de las asignaturas. En próximos ABR debemos hacer más énfasis en este apartado y en las dificultades en los conceptos que puedan tener los alumnos al inicio de las asignaturas. 


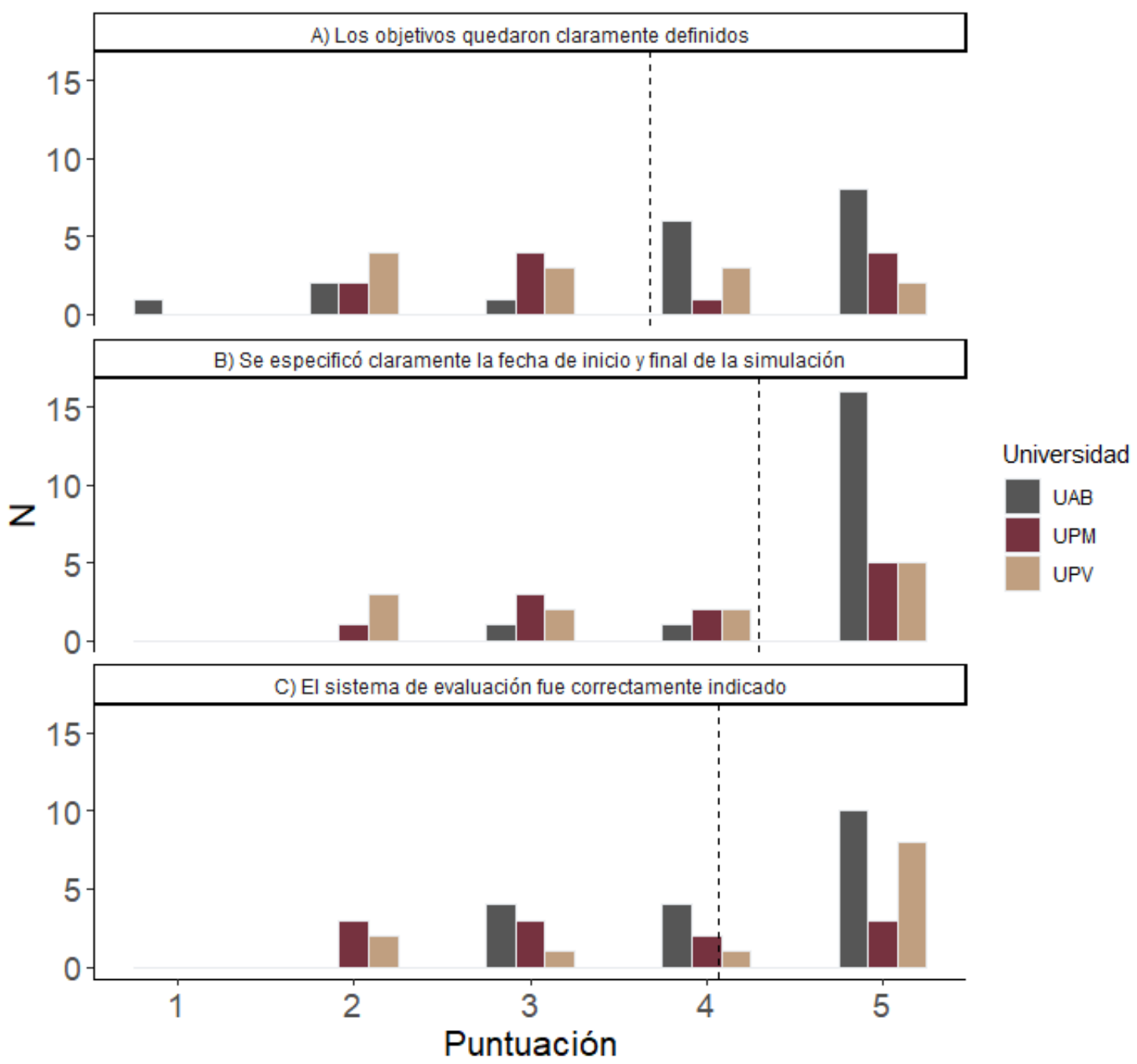

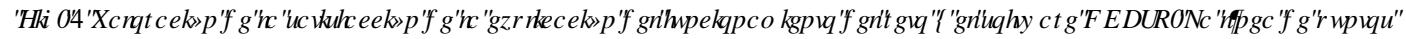

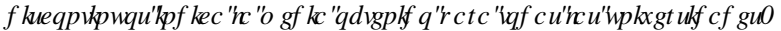

En la Figura 5 se muestran los resultados de la encuesta para las cuestiones relacionadas con la satisfacción del aprendizaje basado en retos a través del software DCBSP. La mayoría de los alumnos cosideraron el reto útil en su aprendizaje con una puntuación media de 4 sobre 5. Así mismo, la satisfacción general y con el programa fue mayor de 4 . El grado de dificultad del reto obtuvo una puntuación media de 3.4 sobre cinco. La mayoría de los estudiantes manifestó que la mayor dificultad la encontraron al inicio cuando no dominaban algunos conceptos de genética, en línea con la puntuación obtenida en la explicación de los objetivos (Figura 4). No se encontraron diferencias entre Universidades. 


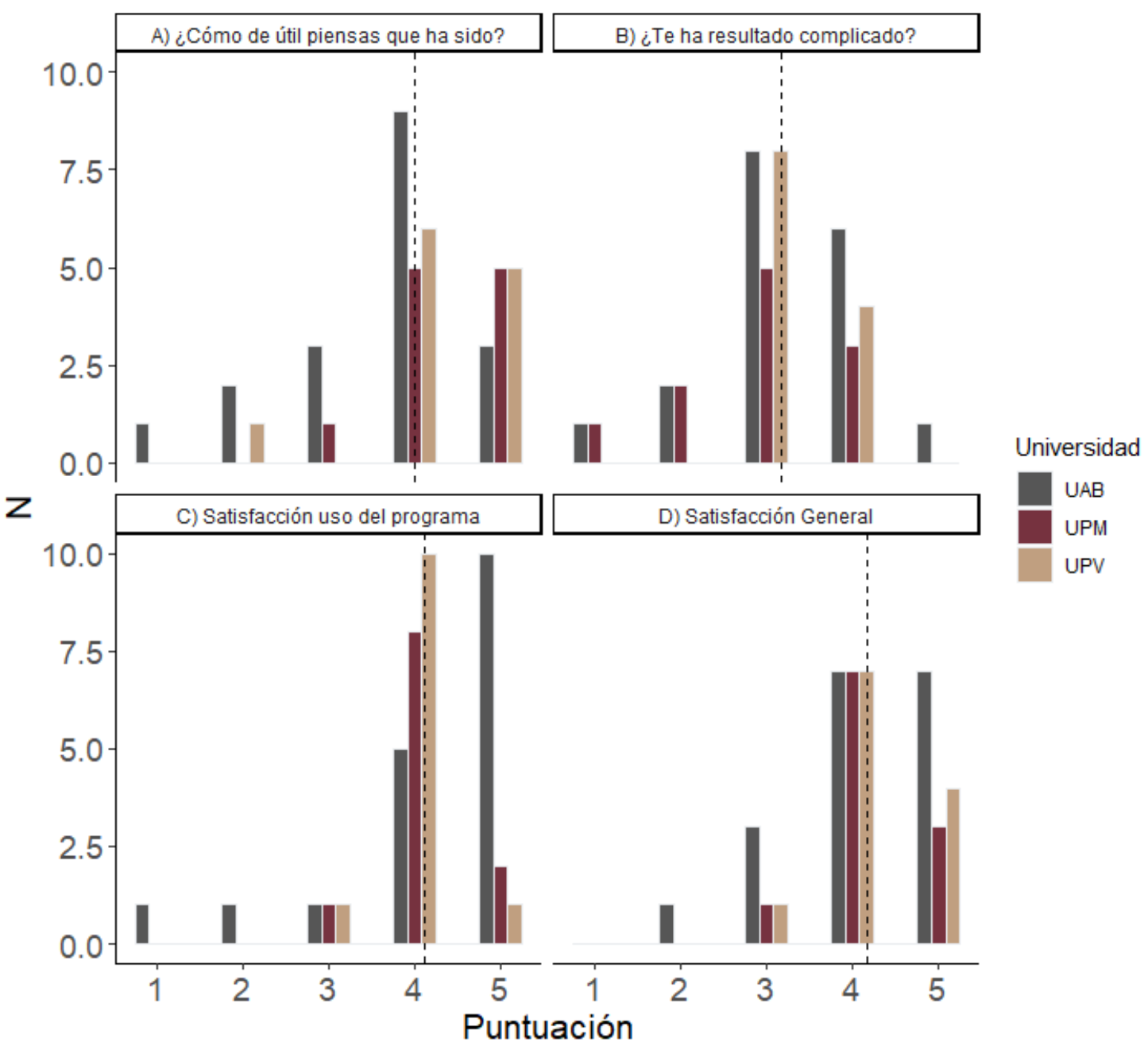

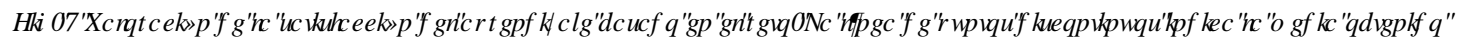
SDWIKRGDQDVXQYYHUGDAHV

La Figura 6 muestra el porcentaje de estudiantes por Universidad que repetiría el reto. El mayor porcentaje se obtuvo en la UPV con un $100 \%$ aunque no hubo diferencias significativas entre Universidades. Este alto porcentaje de alumnos que repetirían el reto indicaría una gran aceptación de este tipo de aprendizaje así como una alta motivación por llevarlo acabo. Por otra parte, la mayoría de los alumnos considera que si lo repitiese mejoraría los resultados, lo que implica que el alumno ha reflexionado sobre los errores cometidos en el programa de mejora animal y considera que ha aprendido a como resolverlos correctamente

Una de las mejoras destacadas por la docencia a través del presente ABR ha sido la gran motivación por parte de los estudiantes a la hora de desarrollar el reto semanalmente, así como el trabajo autónomo que han realizado. Esta percepción se basa tanto en las encuestas realizadas al alumnado, como en las impresiones obtenidas durante las tutorías realizadas semanalmente. 


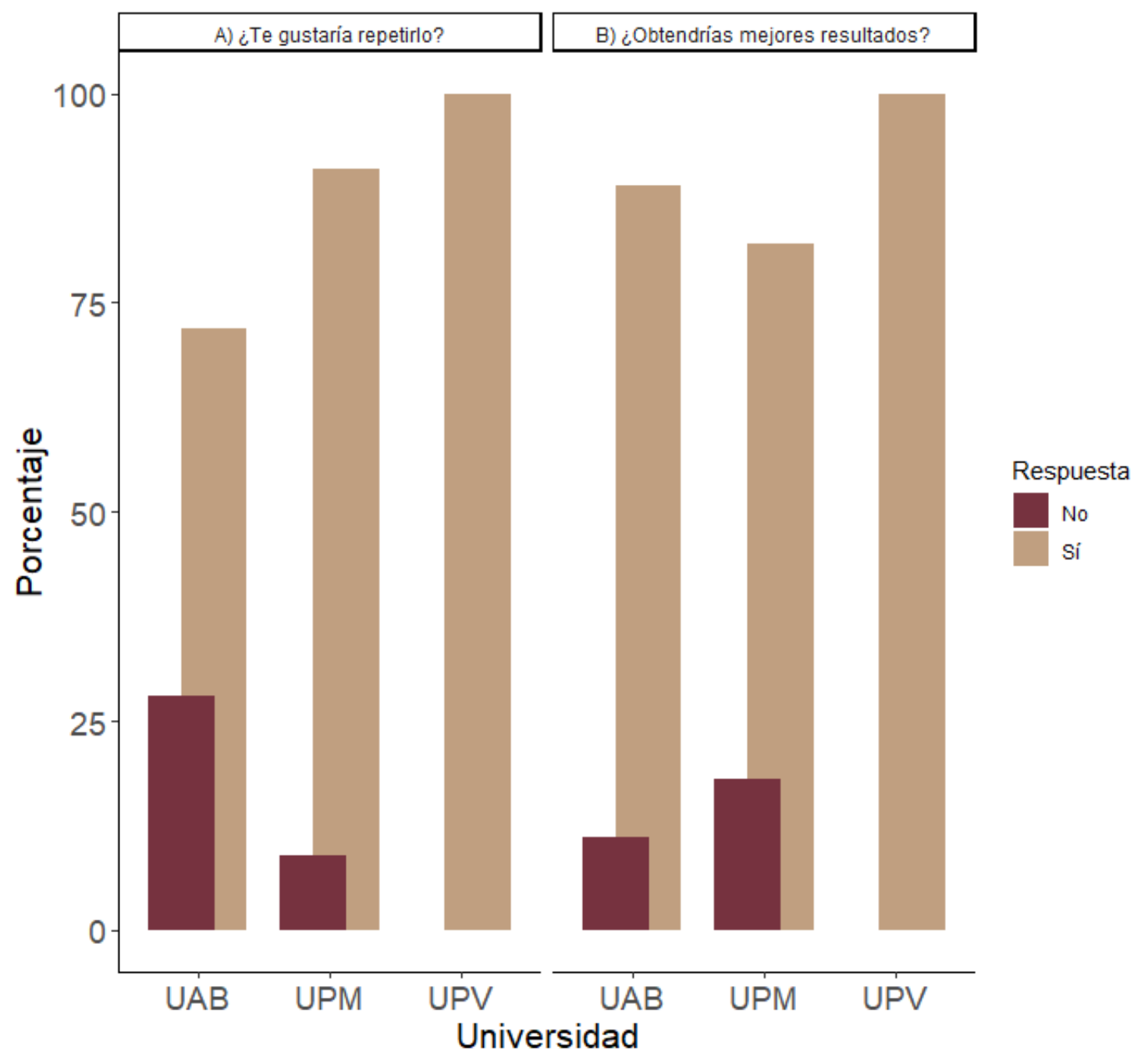

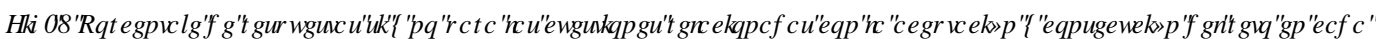
8 QYYHUGDCD

\section{Conclusiones}

La experiencia durante el primer curso de aplicación piloto del ABR con el software DCBSP ha sido muy positiva tanto desde un punto de vista de participación y motivación de los alumnos, como con el desarrollo del pensamiento crítico a través de la aplicación práctica de conceptos teorícos. En general, la implementación del ABR en la asignatura de Mejora Genética Animal fue positiva en las tres Universidades que participaron en el proyecto. No obstante, se deben mejorar algunos aspectos como son la explicación inicial del programa para que sea más sencillo su utilización, así como indicar claramente cuales son los objetivos. Además, se deberá enfatizar en el manual cuál es la información más relevante para la realización correcta del ABR. 


\section{Referencias}

Blank, W. (1997). \$ XUKHQMFIIQWUXFURQ In W.E. Blank \& S. Harwell (Eds.),Tampa, FL: University of South Florida. (ERIC Document Reproduction Service No. ED407586).

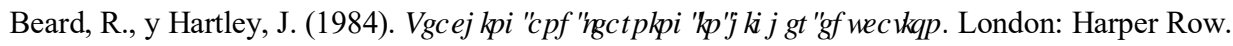
Brown, G., y Atkins, M. (1988). ( IIHFWHНLFКQJШQKJKHUHGXFDMRQ London: Methuen and Co. Buchanan, D.S., Burdit, L.G., Willham, R.L. (1988). "COWGAME: A beef cattle selection simulation program”. $+H H G$ 79: 215

Carlson, J.P. 1985. “Overcoming animal breeding teaching problems”. NACTA Journal 29: 71-73.

Edelson, D.C., Gordin, D.N., Pea, R.D (1999). “Addressing the Challenges of Inquiry-Based Learning Through Technology and Curriculum Design”. 7KH-RXLQDORI UKH / HDQQJ 6FHQFH, 1999, 8(3-4), pp.391-450. ffhal$00190609 f$.

Fletcher, T.S. "Creative thinking in schools: Finding the "just right" challenge for students". * \&7, 34 (2011), pp. $37-$ 42.

Gavin J., K., Elfenbein, H., Staw, B. M. (2017). "The Psychology of Rivalry: A Relationally Dependent Analysis of Competition”. \$FDAP \\RПO DQDJ H HQW RXLQD 53:5 doi.org/10.5465/amj.2010.54533171.

Jou, M., Hung, C. K., y Lai, S. H. (2010).“Application of Challenge Based Learning Approaches in Robotics.Education”. , QUHQDURQDO-RXLQDORIDHFKQRQRJ पDQGF( QJIQHUQJL( GXFDRQ 7(2), 1-42.

Lang, G. (2017). “Agile Learning: Sprinting Through the Semester”. , QIRLP DURQ6। WIP K( GXFDMRQ-RXLQDOVol 15, 3 .

Marti Arias, J. (2010) ( GXFDFyQ IT7FRQRDIDV, Capitulo 4. Libro publicado por el Servicio de Publicaciones Universidad de Cádiz. España.

Leopoldo, A. (2014). "Mipob: un programa de simulación para el aprendizajeen mejora genética animal". \$LFKIRVCH =RRUFQD 63 (244): 665-676. 2014.

Medrano, J.F., Ahmadi, A. and Casellas, J. (2010). "Dairy Cattle Breeding Simulation Program: A simulation program to teach animal breeding principles and practices". - - DUL■6F, 93: 2816-2826.

Piedrafita, J. (2015). "La enseñanza de la Mejora Genética Animal. ¿Qué, cuándo y dónde? ". ,7( \$एIQRLPDFyQ

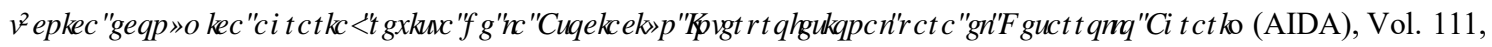
No. 4, 2015, págs. 348-365. 